\title{
Mechanisms behind Overshoots in Mean Cluster Size Profiles in Aggregation-Breakup Processes
}

\author{
Ramiar Sadegh-Vaziri ${ }^{\mathrm{a}}$, Kristin Ludwig ${ }^{\mathrm{b}}$, Kai Sundmacher ${ }^{\mathrm{b}, \mathrm{c}}$, Matthaus U. \\ Babler $^{\mathrm{a}, *}$ \\ ${ }^{a}$ Department of Chemical Engineering, KTH Royal Institute of Technology, SE-10044 \\ Stockholm, Sweden \\ ${ }^{b}$ Max Planck Institute for Dynamics of Complex Technical Systems, Process Systems \\ Engineering, Sandtorstr. 1, 39106 Magdeburg, Germany. \\ ${ }^{c}$ Otto von Guericke University Magdeburg, Process Systems Engineering, \\ Universitätsplatz 2, 39106 Magdeburg, Germany.
}

\begin{abstract}
Aggregation and breakup of small particles in stirred suspensions often shows an overshoot in the time evolution of the mean cluster size: Starting from a suspension of primary particles the mean cluster size first increases before going through a maximum beyond which a slow relaxation sets in. Such behavior was observed in various systems, including polymeric latices, inorganic colloids, asphaltenes, proteins, and, as shown by independent experiments in this work, in the flocculation of microalgae. This work aims at investigating possible mechanism to explain this phenomenon using detailed population balance modeling that incorporates refined rate models for aggregation and breakup of small particles in turbulence. Four mechanisms are considered: (1) restructuring, (2) decay of aggregate strength, (3) deposition of large clusters, and (4) primary particle aggregation where only aggregation events between clusters and primary particles are permitted. We show that all four mechanisms can lead to an overshoot in the mean size profile, while in contrast, aggregation and breakup alone lead to a monotonic, "S"-shaped size evolution profile. In order to distinguish between the different mechanisms simple protocols based on variations of the shear rate during the aggregationbreakup process are proposed.
\end{abstract}

\footnotetext{
*Corresponding author

Email address: babler@kth.se (Matthaus U. Babler )
} 
Keywords: Population balance modeling, colloidal aggregation, shear aggregation, aggregate breakup, restructuring, flocculation, fractal aggregates, turbulence

\section{Introduction}

Aggregation of particles in the nano and micrometer size range is a common unit operation that finds application in many industrial [1] and environmental processes [2, 3. Major applications are found in the processing of colloidal materials, e.g. latex coagulation in the polymer industry [4; nanoparticle synthesis in flame reactors [5, 6]; flocculation in (waste)water treatment [7] or in the production of microalgae [8, 9], and manufacturing of composites or electrodes [10]. In most of these applications the fluid in which the particles are suspended in is vigorously stirred or in the state of turbulence. Fluid flow in the suspending medium has a distinct influence on the aggregation process. On the one hand, it enhances the rate at which particles collide which results in an increase of the aggregation rate. On the other hand, it also creates hydrodynamic stress acting on the aggregates which causes aggregate breakup and affects the aggregate structure.

The interplay between flow induced aggregation and hydrodynamic breakup can lead to atypical dynamics in the time evolution of the aggregate size distribution. In particular, in certain cases one observes an overshoot in the time evolution of the mean aggregate size, i.e. starting from a suspension of primary particles the mean particle size first increases before passing through a maximum beyond which a slow relaxation is observed. Such behavior was observed in the aggregation of fully destabilized polystyrene colloids in stirred tank [11] and Taylor-Couette reactors [12]; in the flocculation of precipitated calcium carbonate in stirred tank [13, 14] and tubular reactors [15]; in the flocculation of suspended solids from raw waters [16]; in the aggregation of asphaltenes in Taylor-Couette reactors [17]; and in the aggregation of amyloid fibrils [18. In addition, in the appendix we present independent experimental data that shows that overshooting also occurs in the flocculation of microalgae in stirred jars. Notably, while most of these studies used light scattering for measuring the particle size, the phenomenon is also seen when using FBRM [15] or image analysis [16, 17].

The occurrence of a maximum in the time evolution of the mean aggregate size is an intriguing phenomenon whose origins are only partially 
understood [12, 11]. Naively, the observed maximum may be explained by aggregate breakup that "kicks in" once the aggregates have reached a certain size. Aggregate breakup is clearly an important phenomenon in stirred environments, as it controls the stationary size distribution that establishes after a long time. However, breakup alone cannot explain the occurrence of a maximum in the mean aggregate size. Analysis of the time scales of the involved processes reveals that aggregation and breakup are relatively fast with respect to the time scale of the relaxation process that occurs later in the process, which suggest that the aggregate sizes observed during the slow relaxation are the outcome of a balance between fast aggregation and breakup rather than breakup alone.

A refined interpretation relates the overshoot to the restructuring of the aggregates [12, 19, 20]. Restructuring clearly is a valid explanation for the observed overshoot that also received experimental support through the measurement of the scattered light intensity in time [12, 13, 19]. A recent study [21] also provides theoretical support for restructuring, arguing that open aggregates in flows undergo restructuring without any energy costs. However, restructuring is not the only mechanism that leads to an overshoot and other mechanisms might apply too. One such mechanism was proposed by Moussa et al. [11] and corroborated by Caimi et al. [22]. These studies argue that the aggregates get weaker as the stirring proceeds, i.e. due to the repeated breakup and re-aggregation the surfaces of the primary particles that constitute the aggregates get more rough. This results in weaker bonds among the primary particles which translates into weaker aggregates and higher breakup rates. This weakening of the aggregates gives breakup a small gain over aggregation such that in effect, the size resulting from the balance between aggregation and breakup slowly decreases in time.

The two mechanisms mentioned above assign the overshoot to either a gradual decrease in the aggregation rate in the case of restructuring, or to a gradual increase of the breakup rate in case of surface deterioration. However, it is conceivable to also have mechanisms that lead to an overshoot and that rely on aggregation and breakup functions that are constant-in-time. Specifically, one can think of a scenario where aggregates are excluded from the aggregation-breakup dynamics upon reaching a certain size, e.g. they might be removed from the system due to settling, deposition or floatation when becoming large. The removal of aggregates of a certain size disturbs the balance between aggregation and breakup, i.e. in shear aggregation, the large aggregates act as "collectors" of smaller aggregates and primary 
particles. Hence, removing the large aggregates leads to a reduction of the aggregation rate which gives breakup a small gain in controlling the evolution of the aggregate size distribution, which eventually results in a decrease of the mean aggregate size.

Another scenario is the case where aggregation is dominated by reactions with primary particles. The extreme case of such dynamics where only reactions between aggregates and primary particles are permitted was considered by Nicoud et al. [18] to model the aggregation of amyloid fibrils. It was shown that such mechanism indeed leads to a maximum in the evolution of the mean size. The maximum occurred when all primary particles are consumed and the slow relaxation in the later stage of the process was controlled by breakup. However, in the system studied by Nicoud et al. [18] aggregation and breakup was controlled by the Brownian motion of particles. Exploring the dominance of primary particle aggregation in a shear controlled system of colloidal aggregates undergoing aggregation and breakup, to the best of our knowledge, has not been done.

The aim of this work is to explore the four mechanisms mentioned above through detailed numerical simulations based on population balance equations (PBE). The four mechanisms considered are (1) restructuring, (2) decay of aggregate strength, (3) deposition of large aggregates, and (4) dominance of primary particle aggregation. Investigating the four mechanisms using the same PBE model allows for identifying the specific characteristics of each mechanism. We show that all four mechanisms lead to maximum in the time evolution of the mean radius of gyration, while some mechanisms also show a maximum in the evolution of the number-mean aggregate mass. Moreover, we propose simple protocols how to identify the controlling mechanism in lab experiment that only measure the mean aggregate size. The proposed protocols rely on step changes of the stirring speed during the aggregation experiment and measure the response of the system. Step changes in the stirring speed are relatively easy to implement in lab experiments [23, 24] making the proposed protocols readily available. Being able to identify the controlling mechanism that causes the overshoot is important for optimizing aggregation processes and tailoring the produced flocks to fit the requirements of the specific application. 


\section{PBE Modeling}

We consider a spatially homogeneous suspension initially containing primary particles of radius $R_{p}$ at a number concentration of $N_{p}$, resulting in a solid volume fraction of $\phi=\frac{4}{3} \pi R_{p}^{3} N_{p}$. The suspension is subject to turbulent stirring which creates a mean shear rate $G$ within the suspension. Turbulent fluctuations, which mainly affect breakup [25, 26], are assumed to be spatially homogeneous around the mean shear rate, i.e. homogeneous turbulence is assumed. Moreover, the suspension is assumed to be fully destabilized such that particles aggregate upon collision. Within this framework, the size evolution of cluster mass distribution is described by a PBE which with the cluster mass taken as a continuous variable reads as:

$$
\begin{aligned}
\frac{d n(m, t)}{d t}= & \frac{1}{2} \int_{0}^{m} K_{A}\left(m-m^{\prime}, m^{\prime}\right) n\left(m^{\prime}, t\right) n\left(m-m^{\prime}, t\right) d m^{\prime} \\
& -n(m, t) \int_{0}^{\infty} K_{A}\left(m, m^{\prime}\right) n\left(m^{\prime}, t\right) d m^{\prime} \\
& +\int_{m}^{\infty} K_{B}\left(m^{\prime}\right) g\left(m, m^{\prime}\right) n\left(m^{\prime}, t\right) d m^{\prime}-K_{B}(m) n(m, t)
\end{aligned}
$$

where $n(m, t)$ is the cluster mass distribution with $m$ being the cluster mass expressed as a multiple of the primary particle mass, $K_{A}\left(m, m^{\prime}\right)$ is the aggregation rate function, $K_{B}(m)$ is the breakup rate function, and $g\left(m, m^{\prime}\right)$ is the fragment distribution function. The first two terms on the right hand side of Eq. (11) refer to birth, respectively, death of clusters of mass $m$ due to aggregation, while the last two terms refer to birth, respectively, death of cluster of mass $m$ due to breakup.

In the absence of coalescence or sintering, fractals or fractal-like structures are formed when small particles stick together. Here, we use the fractal concept in the common form to relate the aggregate size (expressed through the radius of gyration) to the aggregate mass (expressed as a multiple of the primary particle mass) as:

$$
m=k_{g}\left(R_{g} / R_{p}\right)^{d_{f}}
$$

where $d_{f}$ is the aggregate fractal dimension, $R_{g}$ is the radius of gyration and $k_{g}$ is a prefactor that is commonly taken as $k_{g}=1$. 


\subsection{Aggregation rate}

In this work, we consider the case where aggregation is induced by Brownian motion and fluid shear. For fully destabilized particles these two mechanisms add up linearly [1, 27], resulting in:

$K_{A}\left(m, m^{\prime}\right)=\frac{2 k_{B} T}{3 \mu} \frac{\left(m^{1 / d_{f}}+m^{1 / d_{f}}\right)^{2}}{\left(m m^{\prime}\right)^{1 / d_{f}}}+k_{0} f\left(m, m^{\prime} ; d_{f}\right) G R_{p}^{3}\left(m^{1 / d_{f}}+m^{\prime 1 / d_{f}}\right)^{3}$

where the two terms on the right hand side represents Brownian aggregation and shear aggregation, respectively, with $\mu$ being the viscosity, $k_{0}$ being a numerical prefactor set to $k_{0}=1.3$ [28] if not specified otherwise. Moreover, $f\left(m, m^{\prime} ; d_{f}\right)$ is the collision efficiency which in the present work is described through the porous sphere model of Babler [29]. The latter takes into account the hydrodynamic and colloidal interactions of the colliding clusters which results in a significant correction of the classical Saffman-Turner expression (given by the second term of Eq. (3)). The collision efficiency model requires two parameters to quantify the colloidal interactions, namely a dimensionless Hamaker constant, $N_{F}=0.15 A_{H} / \mu R_{p}^{3} G$, and a dimensionless retardation length, $N_{L}=\lambda_{L} / R_{p}$, which were calculated assuming a London wave length of $\lambda_{L}=100 \mathrm{~nm}$ and a Hamaker constant of $A_{H}=3 \times 10^{-21} \mathrm{~J}$, typical for polystyrene in water [30]. Fig. S1 in the Supplementary Materials shows a contour plot of the collision efficiency $f\left(m, m^{\prime} ; d_{f}\right)$. From this figure it is seen that the collision efficiency exhibits a maximum for the collision of triplets followed by a slow decreases as the aggregate size increases or when the size difference between the colliding aggregates increases.

\subsection{Breakup rate}

Describing breakup within the framework of PBEs is an intriguing and long-standing problem [31, 32, 33, 34]. The often applied power law model provides an adequate phenomenological description [35, 36] but fails in capturing the physical mechanism that controls breakup. On the other hand, the semi-empirical exponential model [37] is based on oversimplified assumptions and may cause stability issues when integrating the PBE [38]. A mechanistic model for breakup in turbulent flow was proposed by Babler et al. [39, 40]. These authors argue that (i) the hydrodynamic stress experienced by an aggregate is highly intermittent due to the turbulence that creates the stress, and that (ii) breakup occurs very fast when the instantaneous hydrodynamic 
stress applied on an aggregate exceeds a critical threshold [41, 42, 43]. The latter assumptions implies that the aggregates behave as brittle materials which holds for particles aggregated in the primary minimum of the attraction potential, i.e. for fully destabilized systems [44].

For small non-inertial aggregates the hydrodynamic stress experienced by an aggregate is proportional to the local energy dissipation rate $\varepsilon$. Hence, the occurrence of breakup is controlled by time it takes for an aggregate to experience a local dissipation that exceeds a threshold dissipation $\varepsilon_{\mathrm{cr}}$, representing the aggregate strength. Denoting this time lag by $\tau_{\varepsilon_{\mathrm{cr}}}$, the breakup rate follows as $K_{\varepsilon_{\mathrm{cr}}}^{B}=\left\langle\tau_{\varepsilon_{\mathrm{cr}}}\right\rangle^{-1}$. In [39] the $\tau_{\varepsilon_{\mathrm{cr}}}$ was measured by following the trajectories of tracer-like aggregates in a direct numerical simulation of the Navier-Stokes equation in a periodic box. The resulting breakup rate as a function of the critical dissipation is shown in Fig. 1-a. The symbols refer to the numerical experiments while the solid line shows a statistical model that, within the model assumptions, is exact for $\varepsilon_{\mathrm{cr}} \gg\langle\varepsilon\rangle$. An empirical parametrization of the latter reads as:

$$
\ln \left(K_{\varepsilon_{\mathrm{cr}}}^{B} / G\right)= \begin{cases}-0.457 x-2.8775, & x \leq-2 \\ -0.00104 x^{4}-0.02284 x^{3}-0.1494 x^{2} & x>-2 \\ -0.8138 x-3.160 & \end{cases}
$$

where $x=\ln \left(\varepsilon_{\mathrm{cr}} /\langle\varepsilon\rangle\right)$ is the $\log$ of the dimensionless aggregate strength.

To use Eq. (4) within the PBE model we need to establish a relation between the threshold dissipation $\varepsilon_{\mathrm{cr}}$ and the aggregate mass $m$. Sonntag and Russel [42] and others [45, 46, 31, 47] measured the maximum aggregate size that can withstand a certain hydrodynamic stress in various flow devices. It was found that the aggregate size that can withstand a given hydrodynamic stress decreases as a power law, i.e.

$$
R_{g} \sim \sigma^{-p}
$$

where $\sigma$ is the hydrodynamic stress. The exponent $p$ varied in between 0.35 [31] and 0.60 [47], depending on the aggregate fractal dimension [48]. Recalling that the hydrodynamic stress is related to the local dissipation through $\sigma \sim \mu\left(\varepsilon_{\mathrm{cr}} / \nu\right)^{1 / 2}$ while the aggregate mass is related to the aggregate size as $R_{g} \sim m^{1 / d_{f}}$, we can re-write Eq. (5) as:

$$
\varepsilon_{\mathrm{cr}} \sim \nu m^{-2 /\left(d_{f} p\right)}
$$


respectively,

$$
\frac{\varepsilon_{\mathrm{cr}}}{\langle\varepsilon\rangle} \sim G^{-2} m^{-2 /\left(d_{f} p\right)},
$$

where $G=(\langle\varepsilon\rangle / \nu)^{1 / 2}$ is the mean shear rate. Eq. (7) provides the basic strength relation that gives the critical dissipation required to break an aggregate of mass $m$. Introducing a proportionality factor $C \sim G^{-2}$ puts Eq. (7) in a convenient form to be used in combination with Eq. (4):

$$
\varepsilon_{\mathrm{cr}} /\langle\varepsilon\rangle=C m^{-2 /\left(d_{f} p\right)}
$$

where the dimensionless aggregate strength parameter $C$ is inversely proportional to the mean shear rate squared. With reference to Soos et al. [45] who in their experiments found $\left\langle R_{g}\right\rangle / \mu \mathrm{m} \approx 25.2(\sigma / \mathrm{Pa})^{-0.5}$ for aggregates with $d_{f} \approx 2.7$ and $R_{p} \approx 0.405 \mu \mathrm{m}$, a typical value for the strength parameter is $C \approx\left(1.5 \times 10^{13} \mathrm{~s}^{-2}\right) G^{-2}$. However, since the aggregate strength is very sensitive to the material and surface chemistry of the primary particles and the coagulant, in the following we use the strength factor $C$ as a free parameter, while the strength exponent is taken as $p=0.5$. The latter presents the average strength exponent for fractals in range $d_{f}=2.4$ to 2.9 [44].

Fig. 1-bc shows the breakup rate function obtained from Eqs. (4) and (8) in the form suitable to use within the PBE given in Eq. (1). As can be seen, the breakup rate is virtually zero for small aggregates but kicks in sharply once the aggregates reach a certain mass. The aggregate mass where breakup kicks in depends strongly on the shear rate, represented through the dimensionless strength parameter $C \sim G^{-2}$. Specifically, Fig. 1-b shows that decreasing the strength parameter (which correspond to increasing the shear rate) results in an increase of the breakup rate and an earlier onset of breakup. Fig. 1 $\mathrm{f}$ c shows the influence of the aggregate fractal dimension on the breakup rate. Increasing $d_{f}$ decreases the breakup rate which is in-line with the notion that more open aggregates are more prone to breakup. For very large aggregates the breakup rate follows a power law $K_{B}(m) \sim m^{1.83 / d_{f}}$.

\subsection{Fragment distribution}

The fragment distribution describes the outcome of breakup, i.e. $g\left(m, m^{\prime}\right) d m$ gives the number of fragments with mass in the interval $(m, m+d m)$ that results from the breakup of a cluster of mass $m^{\prime}$. Motivated by the experimental findings of Saha et al. [25], we consider symmetric binary breakup 



Figure 1: Breakup rate employed in this work. (a) Breakup rate as a function of the critical dissipation $\varepsilon_{\mathrm{cr}}$ representing the aggregate strength [39]. Symbols: numerical experiments, solid line: statistical model. (b, c) Breakup rate as a function of aggregate mass, with panel (b) and (c) showing the influence of the aggregate strength parameter an the fractal dimension, respectively. $\langle\varepsilon\rangle$ is the mean energy dissipation rate and $G$ is the mean shear rate.

with the fragment distribution expressed as:

$$
g\left(m, m^{\prime}\right)=(b+1) \frac{2}{m^{\prime}}\left(-\left|\frac{2 m}{m^{\prime}}-1\right|+1\right)^{b}
$$

where $b$ is a precision exponent. Fig. S2 in the Supplementary Materials shows the fragment distribution for different values of $b$. For $b \rightarrow \infty$ the fragment distribution reduces to a peak, i.e. two fragments exactly half the size of the original cluster. In the calculations run here we arbitrarily set $b=8$, resulting in two fragments that differ by less than $25 \%$ in two out of three cases.

\subsection{Numerical Implementation}

Eq. (1) is solved using the fixed pivot method of Kumar and Ramkrishna [49]. In this method, the continuous variable of the system $(m)$ is discretized on a geometric grid with $m_{i+1}=2^{q} m_{i}$, where the grid parameter $q>0$. In our simulations, $q=1 / 3$ was used as the fined grid while $q=1$ was used in the case of coarse grid. The upper limit of the size grid in all simulations is $m_{\max } \approx 10^{12}$, except for the case primary particle aggregation and systems with deposition of large clusters where we considered $m_{\max } \approx 10^{9}$ and $m_{\max } \approx 8 \times 10^{3}$ respectively.

Solving Eq. (1) gives the cluster mass distribution $n(m, t)$, from which the average aggregate size is obtained. In this work, we consider the mean radius of gyration as our principle quantity to evaluate the dynamics of the 
system. In experiments, the latter is readily accessible from light scattering measurements [12, 42, 50], while its relation to the cluster mass distribution is [27, 50]:

$$
\left\langle R_{g}\right\rangle=\left(\frac{\int_{0}^{\infty} n(m, t) m^{2}\left[R_{g}(m)\right]^{2} d m}{\int_{0}^{\infty} n(m, t) m^{2} d m}\right)^{1 / 2}
$$

where $R_{g}(m)$ is the radius of gyration of an aggregate of mass $m$, which is expressed through the strict definition $R_{g}(m)=R_{g, 0} m^{1 / d f}$, with $R_{g, 0}=$ $\sqrt{3 / 5} R_{p}$ being the radius of gyration of the primary particle. In one of the cases considered in this work, we compared our simulations with the experimental data from the literature where the average cluster size was reported as the volume mean cluster sized $d_{[4,3]}$ which is defined as:

$$
d_{[4,3]}=\frac{\int_{0}^{\infty} n(m, t)\left[R_{g}(m)\right]^{4} d m}{\int_{0}^{\infty} n(m, t)\left[R_{g}(m)\right]^{3} d m}
$$

If not specified otherwise, in the numerical calculations performed in this work we consider colloidal particles with a primary particle radius of $R_{p}=$ $100 \mathrm{~nm}$. The shear rate was set to $G=100 \mathrm{~s}^{-1}$ which is a typical value found in stirred tank (cf. Tab. 1 in [51]) or Taylor-Couette [52] reactors used in aggregation studies.

\subsection{Aggregation-breakup dynamics without secondary effects}

We first investigate the case where the aggregates are not affected by the secondary effects discussed in the introduction, i.e. we consider an aggregation-breakup process where the aggregate structure and the aggregate strength, characterised by the fractal dimension $d_{f}$ and the strength parameter $C$, respectively, remain constant in time.

Fig. 2 shows the time evolution of the mean radius of gyration plotted versus a dimensionless time $\tau=t / \tau_{c}$, where $\tau_{c}$ is the characteristic time of aggregation defined as:

$$
\tau_{c}=4 \pi /(3 G \phi)
$$


The normalization by $\tau_{c}$ eliminates the direct effect of the shear rate and the particle number concentration on the aggregate growth. The three panels in Fig. 2 show the influence of the fractal dimension (panel a), the strength parameter that is inversely proportional to the shear rate (panel b), and the solid volume fraction (panel c). In all cases the mean radius of gyration increases with time due to the dominance of aggregation and then relaxes to a plateau. As established in previous works using different rate models [53], the plateau is a result of an equilibrium between aggregation and breakup. In none of the cases an overshoot is observed, i.e. a similar growth pattern is observed under all conditions.

Closer inspection of Fig. 2 - a shows that more open aggregates (small $d_{f}$ ) grow faster than more compact aggregates (large $d_{f}$ ) and reach higher steadystate values. The strong influence of the fractal dimension on the aggregate growth rate is a distinct feature of shear aggregation [54 that can be exploited to infer the aggregate structure from growth rate measurements [27]. The observation that the plateau aggregate size increases with decreasing fractal dimension is due to the stronger influence of $d_{f}$ on the aggregation rate than on the breakup rate.

Fig. 2-b shows the influence of the aggregate strength parameter. The aggregate strength affects mainly the plateau value, and increasing the aggregate strength leads to an increase in the plateau aggregate size. The aggregate growth that takes place at the beginning of the process is not affected by the aggregate strength, and the early evolution of the mean radius of gyration for different values of $C$ coincides up to almost before the steadystate. Also, the time to reach steady-state stays almost unchanged when changing $C$.

The influence of the solid volume fraction is shown in Fig. 2-c. Increasing $\phi$ leads to higher plateau values which is in agreement with experiments [51]. The observed increase of the steady state aggregate size with increasing the solid volume fraction is a direct consequence of the competition between aggregation (that is of order two with respect to the aggregate number concentration) and breakup (that is of order one with respect to the aggregate number concentration). Hence, increasing the aggregate number concentration (by increasing $\phi$ ) strongly enhances the aggregation rate with respect to the breakup rate, which can be exploited to infer characteristics of the breakup rate [53]. Similar to $C$, changing $\phi$ does not change the growth rate of the mean size at the beginning of the process.

Fig. S3 in the Supplementary Material shows the influence of the three 
parameters discussed in Fig. 2 on the steady state aggregate size. Analysis of the steady state aggregate size was conducted in an earlier study [53] using simpler rate expressions for aggregation and breakup. In particular, using a power law model for breakup lead to scaling of the steady state aggregate size with the shear rate $G$ and the solid volume fraction $\phi$. While the former was in good agreement, experiments did not support the scaling with $\phi$. Indeed, keeping $G$ constant and letting $\phi \rightarrow 0$ lead to minimum aggregate size [51]. As shown in Fig. S3, using the more realistic rate functions introduced above qualitatively recovers this behavior.

The analysis presented in Fig. 2] shows that the system parameters affect the steady-state aggregate size, while $d_{f}$ also affects the rate of growth and the time to reach steady-state. More importantly, if the system parameters remain constant in time the aggregate size distribution exhibits no overshoot but instead displays a smooth relaxation to the steady state.

\section{Overshoot in the mean size profile}

\subsection{Restructuring of clusters}

Restructuring is associated with a change in the aggregate fractal dimension $\left(d_{f}\right)$ in time. Specifically, using the scaling of the scattered light intensity as a proxy for $d_{f}$, it was shown experimentally that in certain aggregationbreakup processes $d_{f}$ increases monotonically from an initially low value to a constant plateau value [12, 13, 19]. In the PBE model, $d_{f}$ affects the aggregation rate, the collision efficiency and the breakup rate.

To include the time evolution of the fractal dimension into our PBE model we adopt an approach in which $d_{f}(t)$ follows a predefined function instead of evolving $d_{f}(t)$ with a differential equation as often done in the literature [55, $20,13,50$. The advantage of this approach is that it provides the flexibility to study different restructuring scenarios at a reasonable computational effort. The empirical model for $d_{f}(t)$ is taken as the extended sigmoid function outlined in Fig. S4 in the Supplementary Material. It evolves $d_{f}(t)$ from an initial value $d_{f, 0}$ to a final value $d_{f, \infty}$ following an "S"-shaped curve. The latter consists of two branches, namely a first branch where $d_{f}$ grows exponentially followed by a second branch where $d_{f}$ relaxes exponentially. While the second branch is in accord with the often adopted linear model, i.e.

$\frac{d d_{f}}{d t} \sim\left(d_{f, \infty}-d_{f}\right)$ [55], the first branch reflects the notion that aggregates first have to grow to a certain size before restructuring sets in [50, 56, 57, 

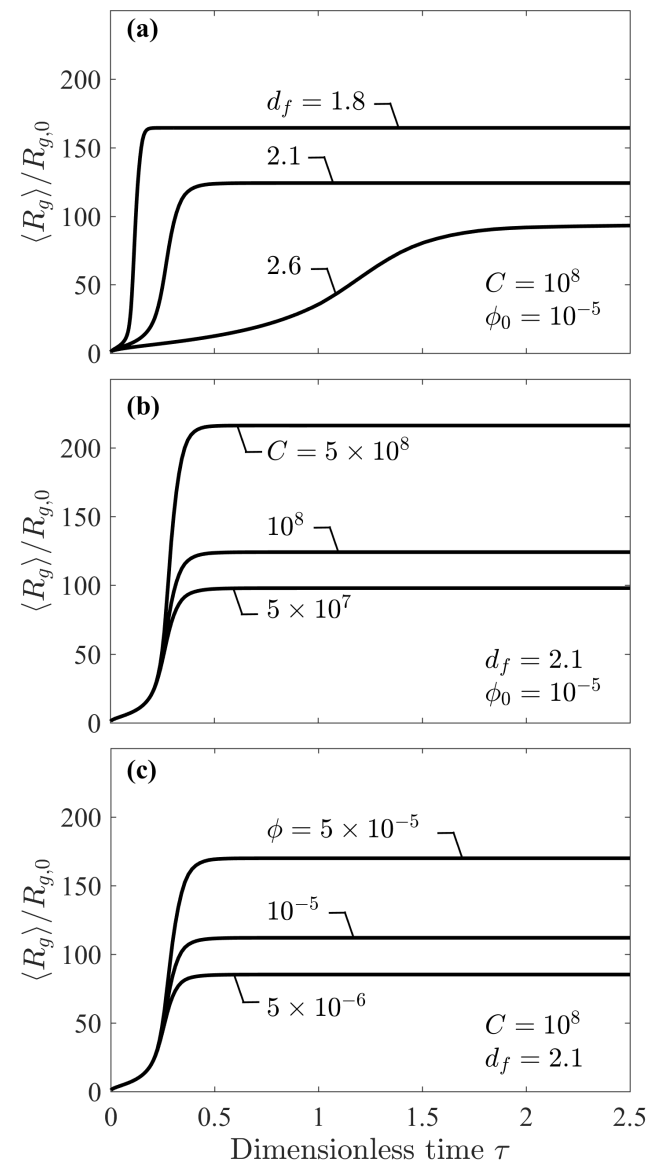

Figure 2: Time evolution of the mean radius of gyration in the absence of secondary effects. The three panels show the influence of the (a) fractal dimension, (b) aggregate strength, and (c) solid volume fraction. 
21. The model for $d_{f}(t)$ is parametrized by two parameters, namely the restructuring rate $\alpha^{\prime}$ characterizing the exponential relaxation to the plateau value, and the inflection point $\tau_{a}^{\prime}$ that specifies the time when restructuring takes place, i.e. $d_{f}(t) \sim e^{-\alpha^{\prime}\left(t-\tau_{2}^{\prime}\right)}$ [see Fig. S4 for a full description of $d_{f}(t)$; a sensitivity analysis [58] is presented in the Supplementary Material]. Below, the parameters are reported in dimensionless form as $\alpha=\alpha^{\prime} \tau_{c}$ and $\tau_{a}=\tau_{a}^{\prime} / \tau_{c}$ where $\tau_{c}$ is the aggregation time given in Eq. (12).

In the following we assume that the aggregates are initially open with $d_{f, 0}=1.8$, which is the typical value for diffusion limited aggregation [59]. Due to breakup and re-aggregation and interactions with the flow [52] [57, 56], the aggregate become more compact and reach a final fractal dimension of $d_{f, \infty}=2.6$, which is the value found by Ehrl et al. 60] for shear aggregation.

Fig. 3 shows the time evolution of the mean radius of gyration (panel a) together with the fractal dimension (panel b). Solid lines show simulations where the fractal dimension is evolving from $d_{f, 0}$ to $d_{f, \infty}$ while dashed lines show simulations with the fractal dimension kept constant at either $d_{f, 0}$ or $d_{f, \infty}$. In the former, we kept constant the restructuring rate $(\alpha=5)$ and varied the time when restructuring occurs, i.e. we considered the cases of early restructuring $\left(\tau_{a}=0.01\right)$, intermediate restructuring $\left(\tau_{a}=0.1\right)$, and late restructuring $\left(\tau_{a}=0.25\right)$. In the case where restructuring occurs at an early time the mean radius of gyration evolves very slowly and the profile exhibits no overshoot. On the other hand, when restructuring occurs at an intermediate or late time, the growth of $\left\langle R_{g}\right\rangle$ is substantially faster and an overshoot is observed. In all cases the mean radius of gyration relaxes to a plateau $\left\langle R_{g}\right\rangle_{\infty} / R_{g, 0} \approx 94$ that is controlled by the final fractal dimension, as indicated by the dashed line that shows the case where $d_{f}$ is constant at $d_{f, \infty}$. The simulations suggest that there is no overshoot when the mean radius of gyration during the restructuring is still smaller than the final plateau value, while on the other hand, if the mean radius of gyration exceeds the final plateau value during the restructuring an overshoot occurs.

In Fig. 4 we explore the evolution of the cluster mass distribution for the case of late restructuring. The three curves show the cluster mass distribution before the peak of the mean size $(t=8 \mathrm{~min}$, indicated by the blue marker in Fig. 3), at the peak ( $t=12 \mathrm{~min}$, orange marker) and at the steady state $(t=50$ min, red marker), with panel (a) showing the mass distribution while panel (b) shows the size distribution. Fig. 4-a shows that heavier clusters are gradually formed as time proceeds, i.e. the mean mass of clusters is monotonically increasing until steady-state is reached. This means that there 



Figure 3: Time evolution of the mean aggregate size in the case of restructuring. (a) Mean radius of gyration and (b) fractal dimension. Solid lines refer to simulations with the fractal dimension evolving from $d_{f, 0}=1.8$ to $d_{f, \infty}=2.6$, while dashed lines refer to simulations with the fractal dimension kept constant at either $d_{f, 0}$ or $d_{f, \infty}$. Here and below, the shear rate is $G=100 \mathrm{~s}^{-1}$ and $R_{p}=100 \mathrm{~nm}$. Markers in panel (a) indicate the times at which the cluster mass distribution is plotted in Fig. 4. 

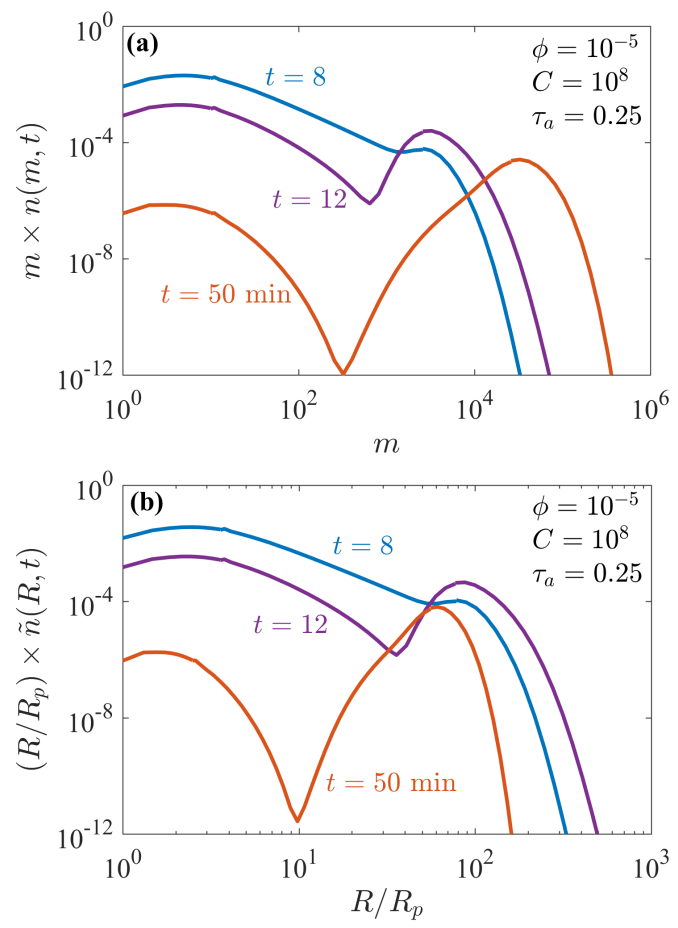

Figure 4: Cluster mass distribution at different times for the case of late restructuring. (a) Cluster mass distribution, (b) cluster size distribution. The latter is readily derived from the mass distribution as $\tilde{n}(R, t)=\left(d_{f} / R_{p}\right) m^{\left(d_{f}-1\right) / d_{f}} \times n(m, t)$, where we made use of $R=R_{p} m^{1 / d_{f}}$.

is no overshoot in the profile of the mean cluster mass versus time. On the contrary, Fig. 4-b shows that the aggregates at the peak are larger than the aggregates at steady state. This confirms that the clusters grow in size up to a point where they start to restructure while the cluster mass constantly increases until reaching a steady state.

To show the feasibility of the proposed mechanism in Fig. 5 we compare our model to the experimental data of Selomulya et al. [55]. The latter conducted aggregation-breakup experiments in a Taylor-Couette reactor using a fully destabilized polystyrene latex. Fig. 5 shows the mean aggregate size and the fractal dimension obtained from the experiments (symbols) together with the model (solid lines). The data suggests that the experiments exhibit late restructuring where the decay of the mean aggregate size in the later stage of the process represents the equilibria between aggregation and 



Figure 5: Time evolution of (a) the mean aggregate size and (b) the fractal dimension as measured by Selomulya et al. [55]. Symbols: experimental data, lines: simulations. Experiments were run in a Taylor-Couette reactor at $G=64 \mathrm{~s}^{-1}$ using a fully destabilized polystyrene latex with $R_{p}=190 \mathrm{~nm}$ at a solid volume fraction of $\phi=3.7 \times 10^{-5}$. Simulation parameters are listed in the figure.

breakup at the given fractal dimension.

\subsection{Decay of aggregate strength}

Repeated breakup and re-aggregation can cause surface alterations of the primary particles contained in the aggregates, which lowers the bond strength between primary particles, making the aggregates weaker [11, 22]. In our model, the decay of the aggregate strength translates into a decrease of the strength parameter $C$ that enters the breakup rate through Eq. (8). To model the decrease of the strength parameter $C$ in time we adapt a similar approach as in the case of restructuring, i.e. we use the sigmoid function outlined in Fig. $\mathrm{S} 4$ to evolve $C(t)$ from an initial value $C_{0}$ to a final plateau value $C_{\infty}$. Using a sigmoid evolution of $C(t)$ instead of, e.g. a monotone relaxation [11] gives us the flexibility to study different scenarios and adds to the numerical stability of the simulation. In the numerical simulations that follow we consider compact aggregates with $d_{f}=2.6$ whose strength 
parameter decreases from $C_{0}=10^{8}$ to $C_{\infty}=10^{7}$.

Fig. 6 shows the time evolution of the mean radius of gyration (panel a) together with the aggregate strength parameter (panel b). The solid lines refer to simulations where the strength parameter is evolving from $C_{0}$ to $C_{\infty}$ while the dashed lines show simulations with the strength parameter kept constant at either $C_{0}$ or $C_{\infty}$. Three cases for the evolution of $C(t)$ are considered, namely fast decay $(\alpha=5)$, intermediate decay $(\alpha=2.5)$, and slow decay $(\alpha=1)$. In all cases the decay sets in at $\tau_{a}=0.5$. Starting the decay of $C(t)$ at earlier times (and at higher values of $C_{0}$ ) does not affect the results, as breakup is insignificant in the early stages of the aggregation process. However, evolving $C(t)$ with the sigmoid function makes the simulation more stable. As can be seen there is no overshoot in the case of fast decay of $C(t)$ while an overshoot is observed for the cases with intermediate and slow decay. In all cases the mean radius of gyration relaxes to a plateau $\left\langle R_{g}\right\rangle / R_{g, 0} \approx 46$ that is controlled by the aggregate strength at the end $\left(C_{\infty}\right)$ as indicated by the dashed line in Fig. 6-a. The simulations suggests that there is no overshoot when the mean aggregate size at the end of the decay of $C(t)$ is still smaller than the final plateau size, as is the case for the fast decay. Inspection of the cluster mass distribution shows that the mean mass of clusters is the largest in the peak. This means that, unlike the case of restructuring, the overshoot is also present in the profile of the mean cluster mass versus time.

To show the applicability of the proposed mechanism, in Fig. 7 we compare our model to the experimental data of Moussa et al. [11. The latter studied the aggregation of a carboxyl polystyrene latex in a stirred tank reactor and showed that the overshoot is caused by a decay in the aggregate strength. Fig. 7 shows the evolution of the mean radius of gyration from one of their experiments (see figure caption for details). For simulating their data we assumed a constant fractal dimension of $d_{f}=2.6$ and set the collision efficiency $f\left(m, m^{\prime} ; d_{f}\right)=1$, in accord with Moussa et al. [11]. Also, since there is no experimental data on the evolution of the aggregate strength in time, the strength decay was fitted to the measured aggregate size assuming the decay starts at the beginning of the experiment $\left(\tau_{a}=0\right)[11$. This leaves us with three parameters $\left(C_{0}, C_{\infty}, \alpha\right)$ to be adjusted. As can be seen from the main panel of Fig. 7 the model describes the experimental data reasonably well. The inset shows the evolution of the strength parameter obtained from the fitted parameters. The strength parameter shows a slow exponential decay, in agreement with Moussa et al. [11. The decay of $C(t)$ is relatively slow 

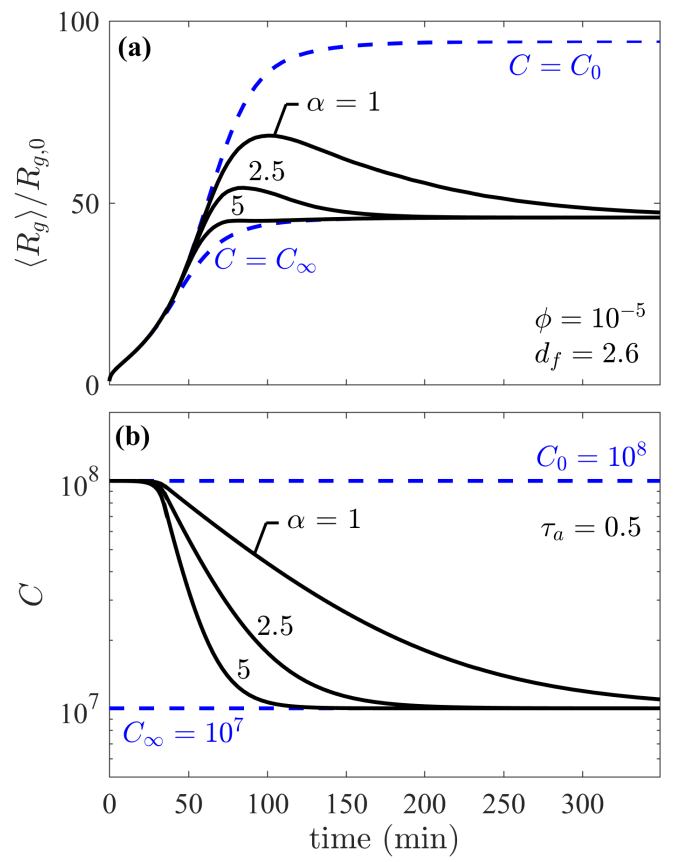

Figure 6: Time evolution of the mean aggregate size for the case of a decaying aggregate strength. (a) Mean radius of gyration and (b) aggregate strength parameter. Solid lines refer to simulations where the aggregate strength parameter decreases from $C_{0}=10^{8}$ to $C_{\infty}=10^{7}$, while dashed lines refer to simulations with the aggregate strength kept constant at $C_{0}$ and $C_{\infty}$. In all simulations, the fractal dimension is constant at $d_{f}=2.6$. 




Figure 7: Time evolution of the mean radius of gyration as measured by Moussa et al. [11. Symbols: experiments, solid lines: simulations. The experiments used a carboxyl polystyrene latex with a primary particle size of $R_{p}=300 \mathrm{~nm}$ at a solid volume fraction of $\phi=2 \times 10^{-5}$; the shear rate was $G=176 \mathrm{~s}^{-1}$. Inset: Evolution of the aggregate strength parameter $C(t)$ obtained by fitting the model to the data in the main panel. The strength parameter evolved from $C_{0}=2.5 \times 10^{9}$ to $C_{\infty}=2.5 \times 10^{8}$.

compared to the time scales of aggregate growth.

\subsection{Deposition of large aggregates}

As the size of aggregates increases, some aggregates may settle, float or stick to the walls of the reactor due to, e.g. gravitational or inertial effects. This leads to zones with much higher solid volume fractions compared to the bulk of the system. Assuming that the interaction of these zones with the rest of the system is negligible, we can exclude the large aggregates that are in these zones from the aggregation-breakup dynamics that take place in the bulk of the system. To simulate this case, we consider a critical aggregate mass, $m_{c}$, above which the aggregates enter the high density zones and are therefore excluded from the system. In the following the critical aggregate mass is taken as $m_{c}=8 \times 10^{3}$ which, for $d_{f}=1.8$, corresponds to a dimensionless aggregate size of $R_{g} / R_{p} \approx 147$. The value for $m_{c}$ was chosen such that the characteristic time for aggregation and breakup of clusters of mass $m_{c}\left(\tau_{\text {agg }}=\left[K_{A}\left(m_{c}\right) N_{p} / m_{c}\right]^{-1}\right.$ and $\left.\tau_{\text {break }}=\left[K_{B}\left(m_{c}\right)\right]^{-1}\right)$ are of similar magnitude, such that the aggregates deposit before suffering extensive breakup.

Fig. 8 shows the time evolution of the mean radius of gyration (panel a) together with the bulk solid volume fraction (panel b). Solid lines refer to the case where there is deposition of large aggregates while dashed lines 
refer to the case without deposition, i.e. the large aggregates stay in the system. The fractal dimension and the aggregate strength are kept constant at $d_{f}=1.8$ and $C=10^{8}$, respectively. As can be seen, the mean radius of gyration increases up to $t \approx 8 \mathrm{~min}$ at which point some of the aggregates are large enough to pass the critical size. Excluding these aggregates from the system results in a decrease in the solid volume fraction as shown in Fig. 8-b. This loss of aggregates disturbs the balance between aggregation and breakup and leads to an overshoot in the mean radius of gyration. The slow decay of $\left\langle R_{g}\right\rangle$ in the second stage of the process is controlled by the rate at which aggregates grow larger than the critical size.

Fig. 9 shows the cluster mass distribution at $t=10 \mathrm{~min}$ for both the case where there is deposition (solid line) and where there is no deposition, i.e. all aggregates stay in the system (dashed line). The two distribution look similar with the peak at $m \approx 4 \times 10^{3}$ reflecting breakup (The breakup rate is shown by the red curve in Fig. Fig. 9). In the case where there is deposition the distribution has a sharp cut-off at the critical mass, while if there is no deposition aggregates can grow larger. The behavior of the cluster mass distribution shown in Fig. 9 implies that for this mechanism there is also an overshoot in the mean aggregate mass.

\subsection{Primary particle aggregation}

Discrete processes where the growth of an entity (or particle) proceeds through the reaction with another entity (or particle) may show a strong dependency on the size of one of the reacting entities. A key example is radical polymerization where the growth of a polymer chain is dominated by reactions with monomers, while in the colloidal domain aggregation of charged particles may show similar characteristics [61, 18]. Other particulate systems that show a strong dominance of reactions that involve monomers may be found in hetero-aggregation or in wet agglomeration processes [61].

An aggregation-breakup process where aggregation is dominated by reactions with primary particles may exhibit an overshoot in the mean aggregate size, i.e. once the primary particles are consumed (or their concentration gets very low) aggregate growth ceases and the evolution of the aggregate size distribution is controlled by breakup, resulting in a decrease of the aggregate size. In the Supplementary Materials we explore such a mechanism for a simplified situation that assumes linearly size-dependent aggregation and breakup rates. It is shown analytically that an aggregation rate that is restricted to reactions with primary particles indeed leads to a maximum in 

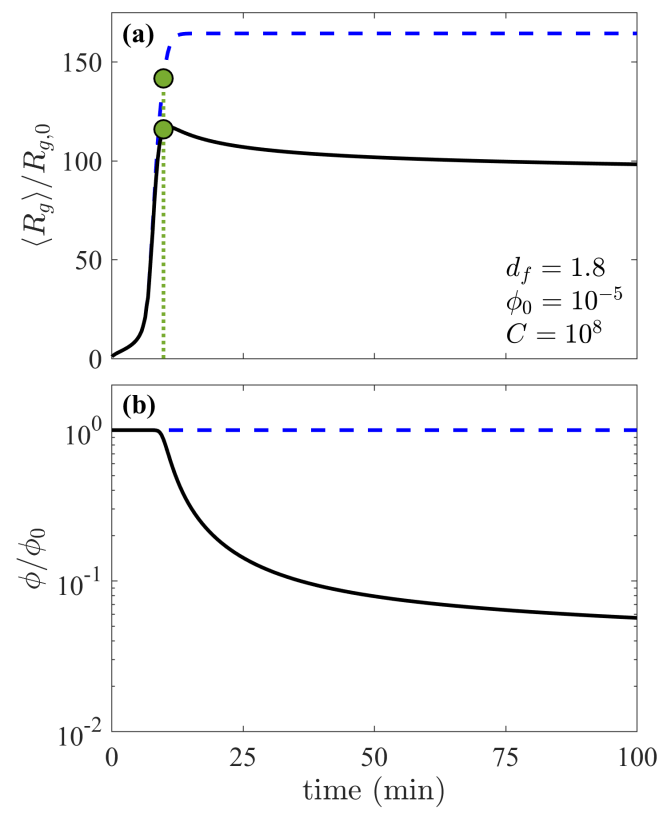

Figure 8: Time evolution of the mean aggregate size for the case of large aggregate deposition. (a) Mean radius of gyration and (b) bulk solid volume fraction. Solid lines refer to simulations with large aggregate deposition while dashed lines refer to simulations where all aggregates remain in the system. The markers in (a) indicate the times at which the cluster mass distribution is shown in Fig. 9 




Figure 9: Cluster mass distribution at $t=10 \mathrm{~min}$ with (black solid line) and without (blue dashed line) large aggregate deposition. The red curve on the right shows the breakup rate $K_{B}(m) / G$.

the time evolution of the mean aggregate size. The overshoot is due to a reduction of the aggregate growth caused by the fading monomer concentration.

In the calculations that follow we aim at exploring the effect of restricted aggregation on a sheared system where aggregation and breakup show a strong size dependency. Restricted aggregation is readily implemented in our PBE model by an aggregation efficiency that reads as:

$$
f\left(m, m^{\prime} ; d_{f}\right)= \begin{cases}\text { finite } & m \text { or } m^{\prime}=1 \\ 0 & \text { otherwise }\end{cases}
$$

where the finite value is obtained from the collision efficiency model used above [29]. Futhermore, in order to observe a pronounced overshoot we set the primary particle radius equal to $R_{p}=500 \mathrm{~nm}$ (as opposed to $100 \mathrm{~nm}$ in other cases considered in this work) and use a coarse grid for the numerical integration of the PBE model.

Fig. 10 shows the time evolution of the mean radius of gyration (panel a) together with the corresponding evolution of the number of primary particles (panel b). The three cases considered refer to aggregates with different strength, as characterized by the strength parameter $C$ that is kept constant in each simulation. For all cases the collision efficiency is given by Eq. (13) while the fractal dimension is kept constant at $d_{f}=1.8$. Increasing the aggregate strength leads to a more pronounced overshoot in the mean size. Stronger aggregates experience lower breakup rates so large clusters have a higher chance of formation. Since large clusters aggregate faster, they con- 

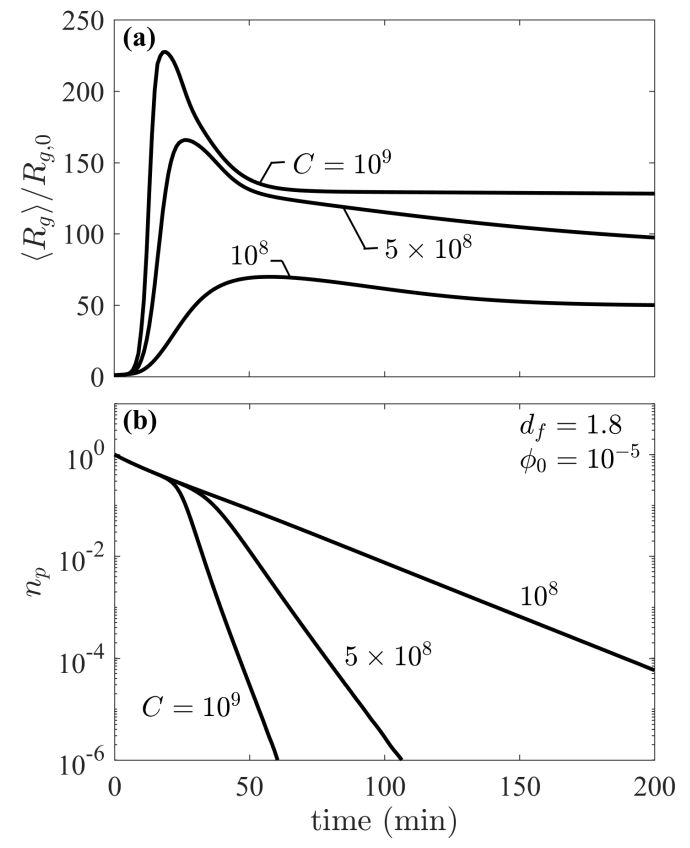

Figure 10: Time evolution of (a) the mean radius of gyration and (b) the number of primary particles for a system governed by primary particle aggregation. The three sets of data refer to different aggregate strength as characterized by the strength parameter $C$.

sume more primary particles. That is why we observe a faster decrease of primary particles in the cases with higher values of $C$, as shown in Fig. 10-b.

To explore the influence of aggregation on the decay of the mean aggregate size in the second stage of the process, in Fig. 11] we present simulations where aggregation is turned off at a specific moment in time. The solid line in Fig. 11 is identical to the case $C=10^{8}$ from Fig. 10, i.e. aggregation is active throughout the process, while the dashed and the dashed-dotted line refer to the cases where aggregation is turned off at the maximum (at $58 \mathrm{~min}$, indicated by the red marker) or at towards the end of the relaxation phase (i.e. at $140 \mathrm{~min}$ ), respectively. As can be seen, when aggregation is turned off at the maximum, the obtained mean size deviates from that of the base case, while when it is turned off towards the end of the relaxation phase the mean sizes almost coincide. This implies that aggregation is only weakly influencing the apparent plateau in the mean size, while it significantly influences the decay after the maximum. Projecting these results to the plot of $n_{p}$ shown in Fig. 11-b, we can conclude that the aggregation rate is negligible when 

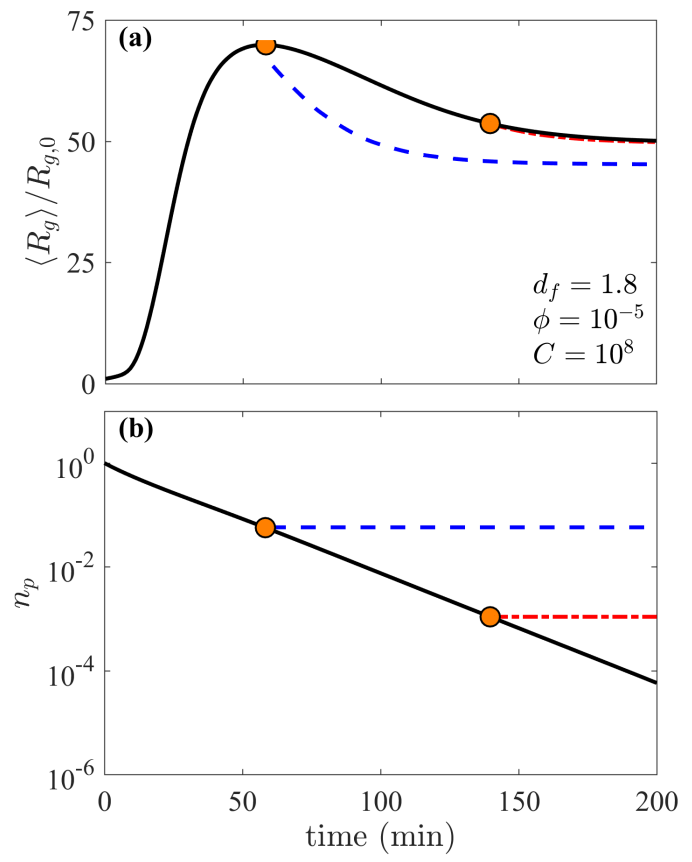

Figure 11: Influence of aggregation on the decay of the mean aggregate size after the maximum for a system governed by primary particle aggregation. The solid line is identical to the case $C=10^{8}$ from Fig. 10, while the dashed line (blue) and the dashed-dotted line (red) refer to the cases where aggregation is turned off at the maximum, respectively, towards the end of the relaxation phase as indicated by the markers.

the number of primary particles goes below $0.1 \%$ of the initial value for the considered system here. Inspection of the cluster mass distribution for primary particle aggregation shows that the mean mass of clusters experience a maximum as well.

\section{Distinguishing among different mechanisms}

Identification of the mechanism that controls the overshoot through direct experimental investigation can be demanding and also may require sophisticated instrumentation [12, 11, 60], especially in the case of complex systems such as wastewaters [16] or microalgae [8]. However, indications from which the controlling mechanism can be deduced can be obtained from measuring the response of the mean aggregate size upon a step change in the shear rate, a technique frequently applied to study the dynamics of aggregation 
and breakup processes [24, 23]. In the following we propose a simple protocol how a step change in the shear rate at a specific moment in time allows for distinguishing among the four mechanisms discussed in this work. The basic principle of the proposed protocol is to compare the time evolution of the mean aggregate size in the case where the shear rate is kept constant with the case where the shear is lowered before and after the maximum in the mean size profile in the original experiment.

Fig. 12 shows the time evolution of the mean aggregate size for the case where the overshoot is controlled by restructuring (black lines) and when it is controlled by a decaying aggregate strength (blue lines). In both cases, the solid lines refer to the situation where the shear rate is kept constant, while the dashed and dashed-dotted lines refer to the situation where the shear rate is reduced to half of its original value before and after the maximum in the original experiment, respectively, as indicated by point 1 and point 2 in Fig. 12 -a. The exact times when the shear rate is lowered were chosen arbitrarily. The simulation parameters were chosen such that the time evolution of the mean aggregate size at constant shear rate is similar for the restructuring case and for the case of a decaying aggregate strength. Since the dependency of restructuring on the shear rate is not known, in our simulation we put restructuring on a halt once the shear rate is lowered. Likewise, we put on halt the decay of the aggregate strength when changing the shear rate.

Fig. 12, a shows the time evolution of the mean aggregate size plotted versus the dimensional time for a shear rate that changes from $G=100$ to $50 \mathrm{~s}^{-1}$. In Fig. 12-b we replot the same data versus the dimensionless time $\tau=t / \tau_{c}$, with $\tau_{c}$ given by Eq. 12 . Notice that due to the dependency of $\tau_{c}$ on the shear rate there are two characteristic aggregation times: one for the interval before the step change in $G$ and one for the interval after the step change, which needs to be accounted for when re-plotting the data in Fig. 12 -b. Normalising the time axis by $\tau_{c}$ cancels out the direct effect of the shear rate on the dynamics of the aggregation process and allows for highlighting the characteristics of the mechanisms leading to an overshoot. In particular, the important characteristic that allows for distinguishing between the two mechanisms is the rate of regrowth upon the step change in $G$. Looking at Fig. 12 -b we see that the rate of re-growth in the case of restructuring (black lines) is substantially different in point 1 and point 2, while in the case of a decaying aggregate strength (blue lines) the rate of re-growth is of similar magnitude in point 1 and point 2 . The reason for this is that reducing the shear rate leads to a drastic reduction of the breakup rate which gives 
aggregation the overhand over breakup. Due to the strong dependency of the aggregation rate on the fractal dimension the restructured aggregates grow substantially slower in point 2 than the more open aggregates in point 1 . In the case of a decaying aggregate strength the fractal dimension and therefore the aggregation rate are not affected. Accordingly, similar growth rates are observed in point 1 and point 2. Such distinguishing was applied by Moussa et al. [11] to relate the overshoot to a decaying aggregate strength.

Fig. 13 shows the results when applying the step change of the shear rate to the case where the overshoot is controlled by deposition of large clusters. The different lines in Fig. 13 have the same meaning as in Fig. 12. The regrowth of the mean aggregate size is similar in the two situations where the step change in $G$ is applied before (point 1) or after (point 2) the maximum of the original experiment, hence, the behavior of the re-growth is similar to the case of a decaying aggregate strength. However, the difference to the latter is that in the case of large aggregate deposition, reducing the shear rate once the apparent plateau is reached results in a re-growth followed by a second overshoot, as shown by the dashed-dotted line in Fig. 13. The reason for this second overshoot is that reducing the shear rate allows for the aggregates to grow to sizes that are prone to deposition. This deposition again disturbs the balance between aggregation and breakup resulting in the second overshoot observed in Fig. 13. (It is worthwhile to add that a second overshoot is unlikely to occur in the case of a decaying aggregate strength: once the original experiment reaches the plateau the weakening of the aggregates gets saturated and it is unlikely that a reduction in the shear rate will result in further weakening, hence, observing a second overshoot upon lowering the shear rate in the very late stage of the experiment indicates to deposition or, e.g. settling caused by the lower stirring speed).

Finally, the response of step changes in the shear rate for a system controlled by primary particle aggregation is shown in Fig. 14. Interestingly, primary particle aggregation shows similar characteristics as the case of restructuring (black lines in Fig. 12), i.e. the re-growth at an early stage is substantially faster than the re-growth at a late stage, as indicated by the slopes $s_{1}$, respectively, $s_{2}$. However, applying the step change at a sufficiently late time (point 3 in Fig. 14) will lead to a pattern where the mean size exhibits negligible re-growth and, instead, stays apparently constant. This apparently constant aggregate size that establishes upon lowering the shear rate is due to the absence of primary particles that would be required for the re-growth. 
Table 1: Distinguising the different overshoot mechanisms

\begin{tabular}{lcc}
\hline Mechanism & Re-grow rates ${ }^{\mathrm{a}}$ & Late stage re-grow pattern \\
\hline Restructuring of clusters & $s_{1}>s_{2}$ & Increase-relaxation \\
Decay of aggregate strength & $s_{1} \approx s_{2}$ & Increase-relaxation \\
Deposition of large clusters & $s_{1} \approx s_{2}$ & Overshoot \\
Primary particle aggregation & $s_{1}>s_{2}$ & Overshoot/decay \\
\hline${ }^{\mathrm{a}} s_{1}$ and $s_{2}$ denote the re-growth rates for a step change in the shear \\
\multicolumn{2}{c}{ rate before and after the maximum in the mean aggregate size. }
\end{tabular}

The results of the analysis to identify the dominating mechanism in a system exhibiting an overshoot are summarized in Table 1. The two characteristics, i.e. the re-grow rates $s_{1}$ and $s_{2}$ and the pattern of the re-growth for a late change in the shear rate, are not the same for any two mechanisms. This provides us the protocol to distinguish among the different mechanisms behind the observed overshoot.

\section{Conclusion}

Many aggregation-breakup processes show an overshoot in the time evolution of the mean cluster size, including latex coagulation, protein aggregation, flocculation of inorganics, asphaltenes aggregation, and as shown in this work, flocculation of microalgea. Aggregation and breakup alone cannot explain this phenomena, i.e. the time evolution of cluster size distribution governed by aggregation and breakup always describes a monotone relaxation to a steady state that is controlled by the shear rate, the solid volume fraction, the fractal dimension and the aggregate strength.

Four mechanisms were considered to investigate the occurrence of an overshoot. Restructuring towards more dense clusters leads to an overshoot when it occurs at a late stage of the process while, on the other hand, early restructuring does not show an overshoot. Hence, restructuring does not per se lead to an overshoot. Similar observation were made for the case of a decaying aggregate strength which shows an overshoot when the decay occurs at a late stage. If the decay happens at an early stage of the process no overshoot is observed. As restructuring is more likely to occur at an early stage (growing aggregates need to restructure in order to not to suffer breakup) while strength decay is more likely to occur at a late stage (at a later stage the aggregates will have experienced many breakup/re-aggregation events) we might argue 



Figure 12: Time evolution of the mean radius of gyration for systems controlled by restructuring (black line) and a decaying aggregate strength (blue lines). The dashed and dashed-dotted lines show the response of the system upon lowering the shear rate to half of its original value. The markers indicate the times when the step change in shear rate are applied. Panel (a) shows the data plotted versus dimensional time, while panel (b) shows the same data versus the dimensionless time $\tau=t / \tau_{c}$. The parameters for the system with restructuring are $k_{0}=0.22, C=10^{8}, d_{f, 0}=1.8, d_{f, \infty}=2.6, \alpha=1$, and $\tau_{a}=0.5$. The parameters for the system with strength decay are $k_{0}=1.3, f=1, d_{f}=2.6, C_{0}=10^{8}$, $C_{\infty}=3.3 \times 10^{7}, \alpha=2.5$, and $\tau_{a}=0.5$. The shear rate varies from $G=100$ to $50 \mathrm{~s}^{-1}$.

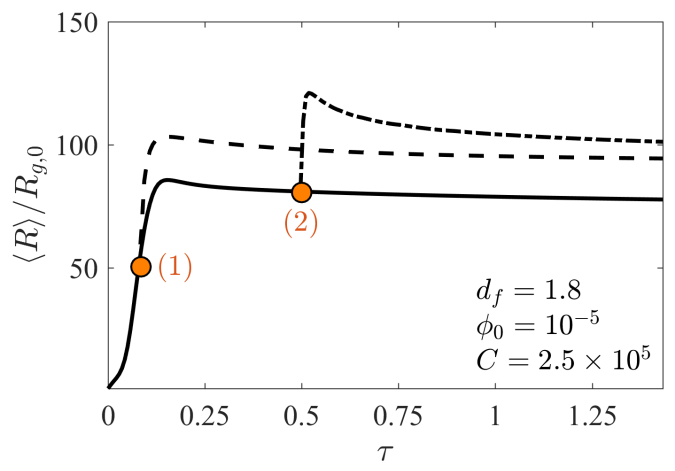

Figure 13: Time evolution of the mean radius of gyration for a system controlled by large aggregate deposition, together with its response upon lowering the shear rate. Lines and markers have the same meaning as in Fig. 12. 




Figure 14: Time evolution of the mean radius of gyration for a system controlled by primary particle aggregation. Lines and markers have the same meaning as in Fig. 12. The slopes $s_{1}$ and $s_{2}$ indicate the rate of regrowth upon a step change in the shear rate.

that strength decay is the prevailing scenario in many aggregation-breakup processes that show an overshoot.

For the other two mechanisms, we found that deposition of large clusters, in principle, leads always to an overshoot. However, a pronounced overshoot that is detectable in an experiment requires a relatively large mass loss, i.e. a decrease of the solid volume fraction by roughly one order of magnitude. Similarly, primary particle aggregation in principle leads always to an overshoot. However, for the overshoot to be detectable the clusters have to be relatively strong such that they can grow to large sizes. Investigating the pattern and rate of re-growth upon a reduction of the shear rate allows for distinguishing between the four mechanisms.

\section{Acknowledgment}

Financial support from the Swedish Research Council VR (grant no 20126216), the Swedish Gasification Centre consortium, and the Center for Dynamic Systems (CDS) funded by the Federal State Saxony-Anhalt, Germany, is greatly acknowledged.

\section{Appendix: Overshoot in the flocculation of the green microalgae D. salina}

Cell cultures of Dunaliella salina (CCAP 19/18, delivered 2011 by the Culture Collection of Algae and Protozoa, Scotland) were prepared prior to 


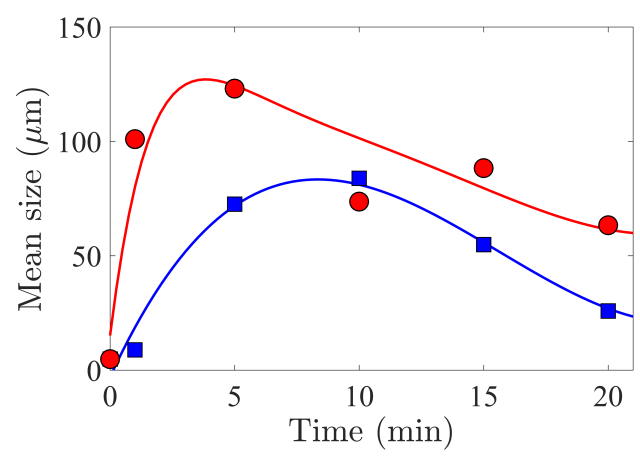

Figure A1: Time evolution of the mean aggregate size in the flocculation of microalga D. salina. The two sets of data refer different flocculants, namely $\mathrm{FeCl}_{3}$ (circles) and $\mathrm{Al}_{2}\left(\mathrm{SO}_{4}\right)_{3}$ (squares). The lines are polynomial interpolations to guide the eye.

flocculation as described in Pirwitz et al. [9]. In short, cultures in a midlogarithmic growth phase were adjusted to a $\mathrm{pH}$ of 7.5 and a cell density of $1.1 \times 10^{7}$ cells $\mathrm{mL}^{-1}$. The flocculation experiments were carried out in a beaker glass using $100 \mathrm{~mL}$ of the previously prepared cell suspension. To induce flocculation, in between 0.4 and $1.0 \mathrm{ml}$ of a flocculant solution was added to the suspension and mixed for $10 \mathrm{~min}$ at $250 \mathrm{rpm}$. The three flocculant solutions were $5 \mathrm{M} \mathrm{NaOH}, 0.1 \mathrm{M} \mathrm{FeCl}_{3} \cdot 6 \mathrm{H}_{2} \mathrm{O}$, and $0.1 \mathrm{M} \mathrm{Al}{ }_{2}\left(\mathrm{SO}_{4}\right)_{3} \cdot 16 \mathrm{H}_{2} \mathrm{O}$, resulting in a flocculant concentration in the suspension of $20 \mathrm{mM} \mathrm{NaOH}$, $1.0 \mathrm{mM} \mathrm{FeCl}_{3}$, or $0.6 \mathrm{mM} \mathrm{Al}_{2}\left(\mathrm{SO}_{4}\right)_{3}$, respectively. After the mixing, the stirring was reduced to $50 \mathrm{rpm}$ and samples of the aggregated cells were taken after $1,5,10,15$ and $20 \mathrm{~min}$ flocculation time $\left(t_{F}\right)$. The samples were analyzed by laser diffraction using a Mastersizer 2000 (Malvern Instruments Ltd, England) instrument. To consider impurities in the medium, a background measurement was done using the pure culture medium. The stable single cell culture was used as control. Fig. A1 shows the mean aggregate size $d_{50}$ versus time for the case of $\mathrm{FeCl}_{3}$ and $\mathrm{Al}_{2}\left(\mathrm{SO}_{4}\right)_{3}$. The time evolution of the mean aggregate size shows an clear overshoot with the maximum occurring after a flocculation time of 5 and $10 \mathrm{~min}$ for the two flocculants, respectively. Using $\mathrm{NaOH}$ as flocculant lead to very large flocks and the no overshoot was detected. The underlying data is reprinted in Tab. S2. As the experiments are the result of an independent preliminary study we do not evaluate the data in terms of the models discussed in this work. 


\section{References}

[1] K.A. Kusters, J.G. Wijers, and D. Thoenes. Aggregation kinetics of small particles in agitated vessels. Chem. Eng. Sci., 52(1):107-121, 1997. doi: 10.1016/S0009-2509(96)00375-2. URL https://doi.org/ 10.1016/S0009-2509(96)00375-2.

[2] A.B. Burd and G.A. Jackson. Particle aggregation. Annu. Rev. Mar. Sci., 1:65-90, 2009. doi: 10.1146/annurev.marine.010908.163904. URL https://doi.org/10.1146/annurev.marine.010908.163904.

[3] D.C. Fugate and C.T. Friedrichs. Controls on suspended aggregate size in partially mixed estuaries. Estuar. Coast. Shelf Sci., 58(2):389-404, 2003. doi: 10.1016/S0272-7714(03)00107-0. URL https://doi.org/ 10.1016/S0272-7714(03)00107-0.

[4] A. Vaccaro, J. Sefcik, H. Wu, M. Morbidelli, J. Bobet, and C. Fringant. Aggregation of concentrated polymer latex in stirred vessels. AIChE J., 52(8):2742-2756, 2006. doi: 10.1002/aic.10843. URL http://dx.doi. org/10.1002/aic.10843.

[5] L. de Martín, W.G. Bouwman, and J.R. van Ommen. Multidimensional nature of fluidized nanoparticle agglomerates. Langmuir, 30(42):1269612702, 2014. doi: 10.1021/la502987e. URL http://dx.doi.org/10. $1021 / 1 a 502987$ e.

[6] H.K. Kammler, L. Mädler, and S.E Pratsinis. Flame synthesis of nanoparticles. Chem. Eng. Technol., 24(6):583-596, 2001. doi: 10.1002/1521-4125(200106)24:6〈583::AID-CEAT583〉3.0. CO;2-H. URL http://dx.doi.org/10.1002/1521-4125(200106)24: 6<583: :AID-CEAT583>3.0.CO;2-H.

[7] J. Ducoste. A two-scale PBM for modeling turbulent flocculation in water treatment processes. Chem. Eng. Sci., 57(12):2157-2168, 2002. doi: 10.1016/S0009-2509(02)00108-2. URL https://doi.org/ 10.1016/S0009-2509(02)00108-2.

[8] D. Vandamme, I. Foubert, and K. Muylaert. Flocculation as a low-cost method for harvesting microalgae for bulk biomass production. Trends Biotechnol., 31(4):233-239, 2013. doi: 10.1016/j.tibtech.2012.12.005. URL https://doi.org/10.1016/j.tibtech.2012.12.005. 
[9] K. Pirwitz, L. Rihko-Struckmann, and K. Sundmacher. Comparison of flocculation methods for harvesting Dunaliella. Bioresource Technol., 196:145-152, 2015. doi: 10.1016/j.biortech.2015.07.032. URL https: //doi.org/10.1016/j.biortech.2015.07.032.

[10] Ricardo MF Fernandes, Matat Buzaglo, Oren Regev, István Furó, and Eduardo F Marques. Mechanical agitation induces counterintuitive aggregation of pre-dispersed carbon nanotubes. Journal of colloid and interface science, 493:398-404, 2017.

[11] A.S. Moussa, M. Lattuada, B. Ó. Conchúir, A. Zaccone, M. Morbidelli, and M. Soos. Flow-induced aggregation and breakup of particle clusters controlled by surface nanoroughness. Langmuir, 29(47):14386-14395, 2013. doi: 10.1021/la403240k. URL http://dx.doi.org/10.1021/ la403240k

[12] C. Selomulya, G. Bushell, R. Amal, and T.D. Waite. Aggregation mechanisms of latex of different particle sizes in a controlled shear environment. Langmuir, 18(6):1974-1984, 2002. doi: 10.1021/la010702h. URL http://dx.doi.org/10.1021/la010702h.

[13] Y. Sang and P. Englezos. Flocculation of precipitated calcium carbonate (PCC) by cationic tapioca starch with different charge densities. I: experimental. Colloid. Surface. A, 414:512-519, 2012. doi: 10.1016/ j.colsurfa.2012.07.019. URL https://doi.org/10.1016/j.colsurfa. 2012.07.019.

[14] E. Antunes, F.A.P. Garcia, P. Ferreira, A. Blanco, C. Negro, and M.G. Rasteiro. Effect of water cationic content on flocculation, flocs resistance and reflocculation capacity of $\mathrm{PCC}$ induced by polyelectrolytes. Ind. Eng. Chem. Res., 47(16):6006-6013, 2008. doi: 10.1021/ie800326z. URL http://dx.doi.org/10.1021/ie800326z.

[15] A.R. Heath, P.A. Bahri, P.D. Fawell, and J.B. Farrow. Polymer flocculation of calcite: experimental results from turbulent pipe flow. AIChE J., 52(4):1284-1293, 2006. doi: 10.1002/aic.10729. URL http: //dx.doi.org/10.1002/aic.10729.

[16] P. Bubakova, M. Pivokonsky, and P. Filip. Effect of shear rate on aggregate size and structure in the process of aggregation and at steady state. 
Powder Technol., 235:540-549, 2013. doi: 10.1016/j.powtec.2012.11.014. URL https://doi .org/10.1016/j.powtec.2012.11.014.

[17] N.H.G. Rahmani, J.H. Masliyah, and T. Dabros. Characterization of asphaltenes aggregation and fragmentation in a shear field. AIChE J., 49(7):1645-1655, 2003. doi: 10.1002/aic.690490705. URL http://dx . doi.org/10.1002/aic.690490705.

[18] L. Nicoud, S. Lazzari, D. Balderas Barragán, and M. Morbidelli. Fragmentation of amyloid fibrils occurs in preferential positions depending on the environmental conditions. J. Phys. Chem. B, 119(13):4644-4652, 2015. doi: 10.1021/acs.jpcb.5b01160. URL http://dx.doi.org/10. 1021/acs.jpcb.5b01160.

[19] E. Antunes, F.A.P. Garcia, P. Ferreira, A. Blanco, C. Negro, and M.G. Rasteiro. Modelling PCC flocculation by bridging mechanism using population balances: Effect of polymer characteristics on flocculation. Chem. Eng. Sci., 65(12):3798-3807, 2010. doi: 10.1016/j.ces.2010.03. 020. URL https://doi.org/10.1016/j.ces.2010.03.020.

[20] R.I. Jeldres, F. Concha, and P.G. Toledo. Population balance modelling of particle flocculation with attention to aggregate restructuring and permeability. Adv. Colloid Interfac., 224:62-71, 2015. doi: 10.1016/j.cis. 2015.07.009. URL http://dx.doi.org/10.1016/j.cis.2015.07.009.

[21] B.O. Conchuir, Y.M. Harshe, M. Lattuada, and A. Zaccone. Analytical model of fractal aggregate stability and restructuring in shear flows. Ind. Eng. Chem. Res., 53(22):9109-9119, 2014. doi: 10.1021/ie4032605. URL http://dx.doi.org/10.1021/ie4032605.

[22] S. Caimi, A. Cingolani, B. Jaquet, M. Siggel, M. Lattuada, and M. Morbidelli. Tracking of fluorescently labeled polymer particles reveals surface effects during shear-controlled aggregation. Langmuir, 33(49):14038-14044, 2017. doi: 10.1021/acs.langmuir.7b03054. URL http://dx.doi.org/10.1021/acs. langmuir.7b03054.

[23] M. Vlieghe, C. Frances, C. Coufort-Saudejaud, and A. Liné. Morphological properties of flocs under turbulent break-up and restructuring processes. AIChE J., 63(9):3706-3716, 2017. doi: 10.1002/aic.15745. URL http://dx .doi .org/10.1002/aic.15745. 
[24] C. Biggs, P. Lant, and M. Hounslow. Modelling the effect of shear history on activated sludge flocculation. Water Sci. Technol., 47(11): 251-257, 2003.

[25] D. Saha, M.U. Babler, M. Holzner, M. Soos, B. Lüthi, A Liberzon, and W. Kinzelbach. Breakup of finite-size colloidal aggregates in turbulent flow investigated by Three-Dimensional (3D) Particle Tracking Velocimetry. Langmuir, 32(1):55-65, 2015. doi: 10.1021/acs.langmuir. 5b03804. URL http://dx.doi .org/10.1021/acs . langmuir.5b03804.

[26] D.L. Marchisio, M. Soos, J. Sefcik, and M. Morbidelli. Role of turbulent shear rate distribution in aggregation and breakage processes. AIChE J., 52(1):158-173, 2006. doi: 10.1002/aic.10614. URL http://dx.doi. org/10.1002/aic.10614.

[27] M.U. Babler, A.S. Moussa, M. Soos, and M. Morbidelli. Structure and kinetics of shear aggregation in turbulent flows. I. Early stage of aggregation. Langmuir, 26(16):13142-13152, 2010. doi: 10.1021/la1015516. URL http://dx .doi .org/10.1021/la1015516.

[28] P.G. Saffman and J.S. Turner. On the collision of drops in turbulent clouds. J. Fluid Mech., 1(1):16-30, 1956. doi: 10.1017/S0022112056000020. URL https://doi.org/10.1017/ S0022112056000020.

[29] M.U. Babler. A collision efficiency model for flow-induced coagulation of fractal aggregates. AIChE J., 54(7):1748-1760, 2008. doi: 10.1002/ aic.11496. URL http://dx.doi.org/10.1002/aic.11496.

[30] D.F. Evans and H. Wennerstrom. The colloidal domain. Wiley-Vch, 1999.

[31] Y.M. Harshe, M. Lattuada, and M. Soos. Experimental and modeling study of breakage and restructuring of open and dense colloidal aggregates. Langmuir, 27(10):5739-5752, 2011. doi: 10.1021/la1046589. URL http://dx.doi.org/10.1021/la1046589.

[32] J. De Bona, A.S. Lanotte, and M. Vanni. Internal stresses and breakup of rigid isostatic aggregates in homogeneous and isotropic turbulence. J. Fluid Mech., 755:365-396, 2014. doi: 10.1017/jfm.2014.421. URL https://doi.org/10.1017/jfm.2014.421. 
[33] M.L. Eggersdorfer, D. Kadau, H.J. Herrmann, and S.E. Pratsinis. Fragmentation and restructuring of soft-agglomerates under shear. J. Colloid Interface Sci., 342(2):261-268, 2010. doi: 10.1016/j.jcis.2009.10.062. URL https://doi.org/10.1016/j.jcis.2009.10.062.

[34] K. Higashitani, K. Iimura, and H. Sanda. Simulation of deformation and breakup of large aggregates in flows of viscous fluids. Chem. Eng. Sci., 56(9):2927-2938, 2001. doi: 10.1016/S0009-2509(00)00477-2. URL https://doi.org/10.1016/S0009-2509(00)00477-2.

[35] P.T. Spicer and S.E. Pratsinis. Coagulation and fragmentation: universal steady-state particle-size distribution. AIChE J., 42(6):1612-1620, 1996. doi: 10.1002/aic.690420612. URL http://dx.doi.org/10.1002/ aic. 690420612 .

[36] Y.M. Harshe and M. Lattuada. Breakage rate of colloidal aggregates in shear flow through stokesian dynamics. Langmuir, 28(1):283-292, 2012. doi: 10.1021/la2038476. URL http://dx.doi.org/10.1021/ $1 \mathrm{a} 2038476$.

[37] J.C. Flesch, P.T. Spicer, and S.E. Pratsinis. Laminar and turbulent shear-induced flocculation of fractal aggregates. AIChE J., 45(5):11141124, 1999. doi: 10.1002/aic.690450518. URL http://dx.doi.org/10. 1002/aic.690450518.

[38] M.U. Babler, M. Morbidelli, and J. Bałdyga. Modelling the breakup of solid aggregates in turbulent flows. J. Fluid Mech., 612:261-289, 2008. doi: 10.1017/S002211200800298X. URL https://doi.org/10.1017/ S002211200800298X.

[39] M.U. Babler, L. Biferale, and A.S. Lanotte. Breakup of small aggregates driven by turbulent hydrodynamical stress. Phys. Rev. E, 85(2):025301, 2012. doi: 10.1103/PhysRevE.85.025301. URL https://doi.org/10. 1103/PhysRevE. 85.025301.

[40] M.U. Babler, L. Biferale, L. Brandt, U. Feudel, K. Guseva, A.S. Lanotte, C. Marchioli, F. Picano, G. Sardina, A. Soldati, and F. Toschi. Numerical simulations of aggregate breakup in bounded and unbounded turbulent flows. J. Fluid Mech., 766:104-128, 2015. doi: 10.1017/jfm.2015.13. URL https://doi.org/10.1017/jfm.2015.13. 
[41] M.A. Delichatsios and R.F. Probstein. Coagulation in turbulent flow: theory and experiment. J. Colloid Interface Sci., 51(3):394-405, 1975. doi: 10.1016/0021-9797(75)90135-6. URL https://doi.org/10.1016/ 0021-9797(75)90135-6.

[42] R.C. Sonntag and W.B. Russel. Structure and breakup of flocs subjected to fluid stresses: I. Shear experiments. J. Colloid Interface Sci., 113 (2):399-413, 1986. doi: 10.1016/0021-9797(86)90175-X. URL https: //doi.org/10.1016/0021-9797(86)90175-X.

[43] K. Guseva and U. Feudel. Aggregation and fragmentation dynamics in random flows: From tracers to inertial aggregates. Phys. Rev. E., 95(6):062604, 2017. doi: 10.1103/PhysRevE.95.062604. URL https: //doi.org/10.1103/PhysRevE.95.062604.

[44] B.O. Conchuir and A. Zaccone. Mechanism of flow-induced biomolecular and colloidal aggregate breakup. Phys. Rev. E, 87(3):032310, 2013. doi: 10.1103/PhysRevE.87.032310. URL https://doi.org/10.1103/ PhysRevE.87.032310.

[45] M. Soos, L. Ehrl, M.U. Babler, and M. Morbidelli. Aggregate breakup in a contracting nozzle. Langmuir, 26(1):10-18, 2009. doi: 10.1021/ la903982n. URL http://dx.doi.org/10.1021/la903982n.

[46] M. Kobayashi, Y. Adachi, and S. Ooi. Breakup of fractal flocs in a turbulent flow. Langmuir, 15(13):4351-4356, 1999. doi: 10.1021/la980763o. URL http://dx.doi.org/10.1021/la980763o.

[47] D. Saha, M. Soos, B. Lüthi, M. Holzner, A. Liberzon, M.U. Babler, and W. Kinzelbach. Experimental characterization of breakage rate of colloidal aggregates in axisymmetric extensional flow. Langmuir, 30(48): 14385-14395, 2014. doi: 10.1021/la502686b. URL http://dx.doi.org/ $10.1021 / 1 a 502686 \mathrm{~b}$.

[48] A. Zaccone, M. Soos, M. Lattuada, H.a Wu, M.U. Babler, and M. Morbidelli. Breakup of dense colloidal aggregates under hydrodynamic stresses. Phys. Rev. E, 79(6):061401, 2009. doi: 10.1103/PhysRevE. 79.061401. URL https://doi.org/10.1103/PhysRevE.79.061401.

[49] S. Kumar and D. Ramkrishna. On the solution of population balance equations by discretizationi. a fixed pivot technique. Chem. Eng. Sci., 51 
(8):1311-1332, 1996. doi: 10.1016/0009-2509(96)88489-2. URL https : //doi.org/10.1016/0009-2509(96)88489-2.

[50] M. Soos, J. Sefcik, and M. Morbidelli. Investigation of aggregation, breakage and restructuring kinetics of colloidal dispersions in turbulent flows by population balance modeling and static light scattering. Chem. Eng. Sci, 61(8):2349-2363, 2006. doi: 10.1016/j.ces.2005.11.001. URL https://doi.org/10.1016/j.ces.2005.11.001.

[51] A.S. Moussa, M. Soos, J. Sefcik, and M. Morbidelli. Effect of solid volume fraction on aggregation and breakage in colloidal suspensions in batch and continuous stirred tanks. Langmuir, 23(4):1664-1673, 2007. doi: 10.1021/la062138m. URL http://dx.doi.org/10.1021/ $1 \mathrm{a} 062138 \mathrm{~m}$.

[52] V. Oles. Shear-induced aggregation and breakup of polystyrene latex particles. J. Colloid Interface Sci., 154(2):351-358, 1992. doi: 10.1016/0021-9797(92)90149-G. URL https ://doi .org/10.1016/ 0021-9797 (92)90149-G.

[53] M.U. Babler and M. Morbidelli. Analysis of the aggregationfragmentation population balance equation with application to coagulation. J. Colloid Interface Sci., 316(2):428-441, 2007. doi: 10.1016/j.jcis. 2007.08.029. URL https://doi.org/10.1016/j.jcis.2007.08.029.

[54] T.D. Waite, J.K. Cleaver, and J.K. Beattie. Aggregation kinetics and fractal structure of $\gamma$-alumina assemblages. J. Colloid Interface Sci., 241(2):333-339, 2001. doi: 10.1006/jcis.2001.7694. URL https://doi. org/10.1006/jcis.2001.7694.

[55] C. Selomulya, G. Bushell, R. Amal, and T.D. Waite. Understanding the role of restructuring in flocculation: the application of a population balance model. Chem. Eng. Sci., 58(2):327-338, 2003. doi: 10.1016/S0009-2509(02)00523-7. URL https://doi.org/10. 1016/S0009-2509(02)00523-7.

[56] V. Becker and H. Briesen. A master curve for the onset of shear induced restructuring of fractal colloidal aggregates. J. Colloid Interface Sci., 346(1):32-36, 2010. doi: 10.1016/j.jcis.2010.02.015. URL https://doi. org/10.1016/j.jcis.2010.02.015. 
[57] M. Vanni and A. Gastaldi. Hydrodynamic forces and critical stresses in low-density aggregates under shear flow. Langmuir, 27(21):1282212833, 2011. doi: 10.1021/la2024549. URL http://dx.doi.org/10. 1021/la2024549.

[58] A. Varma, M. Morbidelli, and H. Wu. Parametric sensitivity in chemical systems. Cambridge University Press, 2005.

[59] R. Jullien and R. Botet. Aggregation and fractal aggregates. Ann. Telecomm., 41:343-short, 1987.

[60] L. Ehrl, M. Soos, and M. Morbidelli. Dependence of aggregate strength, structure, and light scattering properties on primary particle size under turbulent conditions in stirred tank. Langmuir, 24(7):3070-3081, 2008. doi: 10.1021/la7032302. URL http://dx.doi.org/10.1021/ $1 \mathrm{a} 7032302$.

[61] N. I. Lebovka. Aggregation of charged colloidal particles. In Polyelectrolyte Complexes in the Dispersed and Solid State I, pages 57-96. Springer, 2012. 